Journal of Organometallic Chemistry, 403 (1991) 11-19

Elsevier Sequoia S.A., Lausanne

JOM 21316

\title{
The preparation and crystal structures of sodium and potassium pentamethylcyclopentadienyl pyridine solvates
}

\author{
Gerd Rabe, Herbert W. Roesky *, Dietmar Stalke, Frank Pauer \\ and George M. Sheldrick
}

Institut für Anorganische Chemie der Universität Göttingen, Tammannstraße 4,W-3400

Göttingen (Deutschland)

(Received July 27th, 1990)

\begin{abstract}
The compounds $\left(\mathrm{C}_{5} \mathrm{Me}_{5}\right) \mathrm{Na} \cdot 3 \mathrm{py}(1)$ and $\left(\mathrm{C}_{5} \mathrm{Me}_{5}\right) \mathrm{K} \cdot 2$ py (2) have been prepared and their crystal structures determined at low temperatures. The crystal structure of 1 exhibits a three-legged stool monomer of composition $\left(\mathrm{C}_{5} \mathrm{Me}_{5}\right) \mathrm{Na} \cdot 3 \mathrm{py}$, whereas 2 has a linear zig-zag chain structure of composition $\left[\left(\mathrm{C}_{5} \mathrm{Me}_{5}\right) \mathrm{K} \cdot 2 \mathrm{py}\right]_{\infty}$.
\end{abstract}

\section{Introduction}

The alkali metal salts of pentamethylcyclopentadiene $\left(\mathrm{Cp}^{\star} \mathrm{H}\right)$ are important reagents for attaching $\mathrm{Cp}^{\star}$-groups to metal complexes. The presence of $\mathrm{Cp}^{\star}$-ligands [1] enhances the solubility of transition metal complexes in organic solvents and has also allowed the isolation of Main Group metal compounds in formally low oxidation states [2].

In recent years there has been a growing interest in the solid state structures of alkali metal cyclopentadiene derivatives, but relatively few stable crystalline alkali metal salts of cyclopentadiene derivatives have been isolated and their structures deternined. The crystal structures of $\left[\mathrm{C}_{5} \mathrm{H}_{4}\left(\mathrm{SiMe}_{3}\right)\right] \mathrm{Li} \cdot \mathrm{TMEDA}$ [3], $\left[\mathrm{C}_{5} \mathrm{H}_{2}\left(\mathrm{SiMe}_{3}\right)_{3}\right] \mathrm{Li} \cdot \mathrm{TMEDA}$ [4], $\left[\mathrm{C}_{5} \mathrm{H}_{2}\left(\mathrm{SiMe}_{3}\right)_{3}\right] \mathrm{Li} \cdot$ Chinuclidin [4], $\left[\mathrm{C}_{5} \mathrm{H}_{2}-\right.$ $\left.\left(\mathrm{SiMe}_{3}\right)_{3}\right] \mathrm{Li} \cdot \mathrm{PMDETA}$ [5], $\left(\mathrm{C}_{5} \mathrm{H}_{4} \mathrm{Me}\right) \mathrm{Li} \cdot \mathrm{TMEDA}$ [6], $\left(\mathrm{C}_{5} \mathrm{H}_{5}\right) \mathrm{Na} \cdot \mathrm{TMEDA}$ [7], $\left(\mathrm{C}_{5} \mathrm{H}_{4} \mathrm{COMe}\right) \mathrm{Na} \cdot \mathrm{THF}[8],\left[\mathrm{C}_{5} \mathrm{H}_{4}\left(\mathrm{SiMe}_{3}\right)\right] \mathrm{K}$ [9] and $\left[\mathrm{C}_{5}\left(\mathrm{CH}_{2} \mathrm{C}_{6} \mathrm{H}_{5}\right)_{5}\right] \mathrm{K} \cdot 3 \mathrm{THF}$ [10] are known. It is interesting that no structures of alkali metal $\mathrm{Cp}^{\star}$ salts have been reported. We describe here the preparation and structural determination of the two new alkali metal $\mathrm{Cp}^{\star}$ salts $\left(\mathrm{C}_{5} \mathrm{Me}_{5}\right) \mathrm{Na} \cdot 3 \mathrm{py}(1)$ and $\left(\mathrm{C}_{5} \mathrm{Me}_{5}\right) \mathrm{K} \cdot 2 \mathrm{py}(2)$.

\section{Results and discussion}

Pentamethylcyclopentadiene reacts cleanly with sodium metal and potassium hydride to give the corresponding alkali metal salts in good yield according to 
Table 1

Crystallographic data for 1 and 2

\begin{tabular}{|c|c|c|}
\hline Formula & $\mathrm{C}_{25} \mathrm{H}_{30} \mathrm{~N}_{3} \mathrm{Na}$ & $\mathrm{C}_{20} \mathrm{H}_{25} \mathrm{~N}_{2} \mathrm{~K}$ \\
\hline Formula weight & 395.5 & 332.5 \\
\hline Data collection at $T\left({ }^{\circ} \mathrm{C}\right)$ & -120 & -120 \\
\hline Crystal size (mm) & $0.1 \times 0.2 \times 0.4$ & $0.4 \times 0.4 \times 0.6$ \\
\hline Spacc group & Pbca & $P 2_{1} / c$ \\
\hline$a(\AA)$ & $15.271(1)$ & $11.454(1)$ \\
\hline$b(\AA)$ & $17.456(1)$ & $10.393(2)$ \\
\hline$c(\AA)$ & $17.680(2)$ & $17.252(3)$ \\
\hline$\beta\left({ }^{\circ}\right)$ & 90 & $106.93(1)$ \\
\hline Cell volume $\left(\dot{\AA}^{3}\right)$ & 4713 & 1965 \\
\hline Formula units per cell $Z$ & 8 & 4 \\
\hline Calculated density $\rho\left(\mathrm{M} \mathrm{gm}^{-3}\right)$ & 1.115 & 1.124 \\
\hline Absorption coefficient $\mu\left(\mathrm{mm}^{-1}\right)$ & 0.077 & 0.27 \\
\hline$F(000)$ & 1696 & 712 \\
\hline Measured $2 \theta$-range $\left({ }^{\circ}\right)$ & $8-45$ & $8-50$ \\
\hline Number of measured reflections & 3126 & 3983 \\
\hline Number of unique reflections & 3058 & 3454 \\
\hline Number of observed reflections & 1666 & 2816 \\
\hline \multicolumn{3}{|l|}{ Consistency of equivalent } \\
\hline reflections $\left(R_{\text {int }}\right)$ & 0.0093 & 0.0547 \\
\hline$F>p \sigma(F) ; p=$ & 3 & 3 \\
\hline$R$ & 0.0666 & 0.0427 \\
\hline$w R$ & 0.0608 & 0.0496 \\
\hline Goodness of fit & 1.73 & 1.59 \\
\hline \multicolumn{3}{|l|}{ Weighting factor $g$} \\
\hline$w=1 /\left(a^{2}(F)+\operatorname{abs}(g) F^{2}\right)$ & 0.0003 & 0.0004 \\
\hline Refined parameters & 262 & 283 \\
\hline \multicolumn{3}{|l|}{ Last difference-Fourier synthesis } \\
\hline Highest maximum $\left(\mathrm{e}^{-\AA^{-3}}\right)$ & 0.26 & 0.25 \\
\hline Lowest minimum $\left(\mathrm{e}^{-} \dot{\mathrm{A}}^{-3}\right)$ & 0.32 & 0.23 \\
\hline
\end{tabular}

equations 1 and 2.

$\mathrm{Na}+\mathrm{C}_{5} \mathrm{Me}_{5} \mathrm{H} \underset{-1 / 2 \mathrm{H}_{2}}{\stackrel{\text { Toluene }}{-1}} \mathrm{NaC}_{5} \mathrm{Me}_{5}$

$\mathrm{KH}+\mathrm{C}_{5} \mathrm{Me}_{5} \mathrm{H} \underset{-\mathrm{H}_{2}}{\stackrel{\mathrm{THF}}{\longrightarrow}} \mathrm{KC}_{5} \mathrm{Me}_{5}$

By dissolving the products from reactions 1 and 2 in pyridine and slowly removing the solvent, single crystals of 1 and 2 suitable for X-ray structural analyses were obtained. Crystal data for 1 and 2 are given in Table 1, fractional coordinates in Tables 2 (1) and $3(2)$, and selected interatomic distances and angles in Tables 4 (1) and 5 (2).

The crystal structure of 1 consists of a three-legged stool monomer with the $\mathrm{Cp}^{\star-}$ pentahapto coordinated to sodium together with three molecules of pyridine (Fig. 1). The molecular structure of $\left(\mathrm{C}_{5} \mathrm{Me}_{5}\right) \mathrm{Na} \cdot 3 \mathrm{py}$ shows it to be the first example of a monomeric cyclopentadienyl derivative of sodium. The $\mathrm{Na}-\mathrm{C}$ bond distances (mean

* A reference number with an asterisk indicates a note in the list of references. 
Table 2

Atomic coordinates $\left(\times 10^{4}\right)$ and equivalent isotropic displacement coefficients ${ }^{a}\left(\dot{\mathrm{A}}^{2} \times 10^{3}\right)$ for 1

\begin{tabular}{|c|c|c|c|c|}
\hline & $x$ & $y$ & $z$ & $U_{\text {eq }}$ \\
\hline $\mathrm{Na}(1)$ & $-32(1)$ & $1171(1)$ & $1603(1)$ & $43(1)$ \\
\hline$C(1)$ & $-382(3)$ & $2582(3)$ & $1117(3)$ & $43(2)$ \\
\hline$C(2)$ & $-189(3)$ & $2660(2)$ & $1885(3)$ & $43(2)$ \\
\hline$C(3)$ & $716(4)$ & $2506(3)$ & $1972(3)$ & $44(2)$ \\
\hline$C(4)$ & $1058(3)$ & $2336(3)$ & $1250(3)$ & $47(2)$ \\
\hline$C(5)$ & $379(4)$ & $2385(3)$ & $735(3)$ & $47(2)$ \\
\hline$C(6)$ & $-1265(4)$ & $2748(3)$ & $767(3)$ & $78(3)$ \\
\hline$C(7)$ & $-813(4)$ & $2917(3)$ & $2499(3)$ & $75(2)$ \\
\hline$C(8)$ & $1230(4)$ & $2563(3)$ & $2700(3)$ & $78(3)$ \\
\hline $\mathrm{C}(9)$ & 1991(4) & $2119(3)$ & $1053(3)$ & $78(3)$ \\
\hline$C(10)$ & $456(4)$ & $2268(3)$ & $-114(3)$ & $79(3)$ \\
\hline $\mathbf{N}(1)$ & $1021(3)$ & $161(2)$ & $1283(2)$ & $49(2)$ \\
\hline$C(11)$ & $1663(4)$ & $202(3)$ & $1790(3)$ & $58(2)$ \\
\hline$C(12)$ & $2341(4)$ & $-308(3)$ & $1854(3)$ & $59(2)$ \\
\hline$C(13)$ & $2377(4)$ & $-910(3)$ & 1351(3) & $58(2)$ \\
\hline$C(14)$ & $1732(4)$ & $-964(3)$ & $825(3)$ & $54(2)$ \\
\hline$C(15)$ & $1080(4)$ & $-428(3)$ & $799(3)$ & $50(2)$ \\
\hline $\mathrm{N}(2)$ & $-1399(3)$ & $529(3)$ & $1252(2)$ & $54(2)$ \\
\hline$C(16)$ & $-1435(4)$ & $-212(3)$ & $1081(3)$ & $50(2)$ \\
\hline$C(17)$ & $-2205(4)$ & $-602(3)$ & $957(3)$ & $60(2)$ \\
\hline$C(18)$ & $-2979(4)$ & $-212(4)$ & $1017(3)$ & $65(3)$ \\
\hline$C(19)$ & $-2968(4)$ & $550(4)$ & $1188(3)$ & $67(3)$ \\
\hline$C(20)$ & $-2161(5)$ & $887(3)$ & $1301(3)$ & $62(2)$ \\
\hline $\mathbf{N}(3)$ & $-225(3)$ & $580(2)$ & $2873(2)$ & $50(2)$ \\
\hline$C(21)$ & $-485(4)$ & $983(3)$ & $3458(3)$ & $66(2)$ \\
\hline$C(22)$ & $-731(5)$ & $665(3)$ & $4132(3)$ & $87(3)$ \\
\hline$C(23)$ & $-687(4)$ & $-116(3)$ & $4225(3)$ & $63(2)$ \\
\hline$C(24)$ & $-413(3)$ & $-550(3)$ & $3634(3)$ & $45(2)$ \\
\hline$C(25)$ & $-195(3)$ & $-175(3)$ & $2970(3)$ & $49(2)$ \\
\hline
\end{tabular}

a Equivalent isotropic $U$ defined as one third of the trace of the orthogonalized $U_{i j}$ tensor

$2.68 \AA$ ) in 1 are considerably shorter than those found in $\left(\mathrm{C}_{5} \mathrm{H}_{5}\right) \mathrm{Na} \cdot$ TMEDA [7] $(2.92 \AA)$ and $\left(\mathrm{C}_{5} \mathrm{H}_{4} \mathrm{COMe}\right) \mathrm{Na} \cdot \mathrm{THF}[8](2.82 \AA)$.

The $\mathrm{Na}-\mathrm{Cp}$ (centre) distance is $2.40 \AA$. The mean bond length between the sodium and the pyridine $\mathrm{N}$-atoms is $2.46 \AA$. The ${ }^{23} \mathrm{Na} \mathrm{NMR}$ spectrum of $\left(\mathrm{C}_{5} \mathrm{Me}_{5}\right) \mathrm{Na}$ in $\mathrm{C}_{5} \mathrm{D}_{5} \mathrm{~N}$ solution gives a single broad line $\left(\Delta \nu_{1 / 2}=420 \mathrm{~Hz}\right)$ at $-22.0 \mathrm{ppm}\left[11^{*}\right]$ (Fig. 2). Presumably the relatively large line width arises from large quadrupolar relaxation effects owing to a highly asymmetric environment around the sodium nucleus [12]. In general ${ }^{23} \mathrm{Na}$ NMR line widths are of the order of a few $\mathrm{Hz}$ for solvated sodium cations [13]. The asymmetric environment of sodium in 1 is probably due to the presence of strong contact $\mathrm{Cp}^{\star-} \mathrm{Na}^{+}$ion pairs in solution. Furthermore, it is likely that $\left(\mathrm{C}_{5} \mathrm{Me}_{5}\right) \mathrm{Na} \cdot 3$ py retains its monomeric structure in pyridine solution.

In contrast to 1, the crystal structure of 2 consists of polymeric zig-zag chains of composition $\left[\left(\mathrm{C}_{5} \mathrm{Me}_{5}\right) \mathrm{K} \cdot 2 \mathrm{py}\right]_{\infty}$ (Fig. 3). In addition to two pyridine molecules, each potassium cation in the chain is approximately pentahapto coordinated to two $\mathrm{Cp}^{\star}$-rings. The angle between the cyclopentadienyl ring normals at the potassium is $138^{\circ}$, smaller than the corresponding angle in the analogous polymer of 
Table 3

Atomic coordinates $\left(\times 10^{4}\right)$ and equivalent isotropic displacement coefficients ${ }^{a}\left(\dot{A}^{2} \times 10^{3}\right)$ for 2

\begin{tabular}{lrrrr}
\hline & \multicolumn{1}{l}{$y$} & \multicolumn{1}{l}{$\boldsymbol{z}$} & $U_{\text {eq }}$ \\
\hline $\mathrm{K}(1)$ & $4093(1)$ & $178(1)$ & $7365(1)$ & $30(1)$ \\
$\mathrm{C}(10)$ & $4885(2)$ & $-2362(2)$ & $6777(1)$ & $32(1)$ \\
$\mathrm{C}(1)$ & $4978(3)$ & $-2390(3)$ & $5924(2)$ & $56(1)$ \\
$\mathrm{C}(20)$ & $3884(2)$ & $-2761(2)$ & $7033(1)$ & $33(1)$ \\
$\mathrm{C}(2)$ & $2668(2)$ & $-3215(3)$ & $6490(2)$ & $55(1)$ \\
$\mathrm{C}(30)$ & $4205(2)$ & $-2640(2)$ & $7886(1)$ & $31(1)$ \\
$\mathrm{C}(3)$ & $3436(3)$ & $-2985(3)$ & $8430(2)$ & $51(1)$ \\
$\mathrm{C}(40)$ & $5404(2)$ & $-2152(2)$ & $8152(1)$ & $29(1)$ \\
$\mathrm{C}(4)$ & $6065(3)$ & $-1784(2)$ & $9012(1)$ & $48(1)$ \\
$\mathrm{C}(50)$ & $5825(2)$ & $-1987(2)$ & $7469(1)$ & $29(1)$ \\
$\mathrm{C}(5)$ & $7077(2)$ & $-1538(2)$ & $7477(2)$ & $45(1)$ \\
$\mathrm{N}(1)$ & $2287(2)$ & $143(2)$ & $8227(1)$ & $46(1)$ \\
$\mathrm{C}(11)$ & $2336(3)$ & $221(3)$ & $9003(2)$ & $58(1)$ \\
$\mathrm{C}(12)$ & $1371(3)$ & $-12(3)$ & $9299(2)$ & $57(1)$ \\
$\mathrm{C}(13)$ & $290(3)$ & $-350(3)$ & $8776(2)$ & $63(1)$ \\
$\mathrm{C}(14)$ & $220(3)$ & $-443(5)$ & $7977(2)$ & $100(2)$ \\
$\mathrm{C}(15)$ & $1237(3)$ & $-211(4)$ & $7735(2)$ & $73(1)$ \\
$\mathrm{N}(2)$ & $1955(2)$ & $490(2)$ & $5906(1)$ & $49(1)$ \\
$\mathrm{C}(21)$ & $2361(2)$ & $168(3)$ & $5290(2)$ & $56(1)$ \\
$\mathrm{C}(22)$ & $1887(3)$ & $638(3)$ & $4511(2)$ & $65(1)$ \\
$\mathrm{C}(23)$ & $926(3)$ & $1478(3)$ & $4365(2)$ & $66(1)$ \\
$\mathrm{C}(24)$ & $486(2)$ & $1809(3)$ & $4982(2)$ & $58(1)$ \\
$\mathrm{C}(25)$ & $1020(2)$ & $1297(3)$ & $5740(2)$ & $50(1)$ \\
\hline
\end{tabular}

${ }^{a}$ Equivalent isotropic $U$ defined as one third of the trace of the orthogonalized $U_{i j}$ tensor

Table 4

Selected bond lengths $(\dot{A})$ and angles $\left({ }^{\circ}\right)$ for 1

\begin{tabular}{lclr}
\hline $\mathrm{Na}(1)-\mathrm{C}(1)$ & $2.663(5)$ & $\mathrm{Na}(1)-\mathrm{N}(3)$ & $2.488(4)$ \\
$\mathrm{Na}(1)-\mathrm{C}(2)$ & $2.658(5)$ & $\mathrm{C}(1)-\mathrm{C}(2)$ & $1.397(7)$ \\
$\mathrm{Na}(1)-\mathrm{C}(3)$ & $2.676(5)$ & $\mathrm{C}(1)-\mathrm{C}(5)$ & $1.387(8)$ \\
$\mathrm{Na}(1)-\mathrm{C}(4)$ & $2.701(5)$ & $\mathrm{C}(2)-\mathrm{C}(3)$ & $1.416(8)$ \\
$\mathrm{Na}(1)-\mathrm{C}(5)$ & $2.691(5)$ & $\mathrm{C}(3)-\mathrm{C}(4)$ & $1.410(7)$ \\
$\mathrm{Na}(1)-\mathrm{N}(1)$ & $2.451(5)$ & $\mathrm{C}(4)-\mathrm{C}(5)$ & $1.382(8)$ \\
$\mathrm{Na}(1)-\mathrm{N}(2)$ & $2.450(5)$ & $\mathrm{Na}(1)-\mathrm{Cp}(\mathrm{centre})$ & $2.399(-)$ \\
$\mathrm{C}(1)-\mathrm{Na}(1)-\mathrm{C}(2)$ & $30.4(1)$ & $\mathrm{C}(1)-\mathrm{Na}(1)-\mathrm{N}(2)$ & $99.8(2)$ \\
$\mathrm{C}(1)-\mathrm{Na}(1)-\mathrm{C}(3)$ & $50.2(2)$ & $\mathrm{C}(2)-\mathrm{Na}(1)-\mathrm{N}(2)$ & $114.7(2)$ \\
$\mathrm{C}(2)-\mathrm{Na}(1)-\mathrm{C}(3)$ & $30.8(2)$ & $\mathrm{C}(3)-\mathrm{Na}(1)-\mathrm{N}(2)$ & $145.5(2)$ \\
$\mathrm{C}(1)-\mathrm{Na}(1)-\mathrm{C}(4)$ & $49.6(2)$ & $\mathrm{C}(4)-\mathrm{Na}(1)-\mathrm{N}(2)$ & $144.2(2)$ \\
$\mathrm{C}(2)-\mathrm{Na}(1)-\mathrm{C}(4)$ & $50.4(2)$ & $\mathrm{C}(5)-\mathrm{Na}(1)-\mathrm{N}(2)$ & $114.5(2)$ \\
$\mathrm{C}(3)-\mathrm{Na}(1)-\mathrm{C}(4)$ & $30.4(2)$ & $\mathrm{N}(1)-\mathrm{Na}(1)-\mathrm{N}(2)$ & $99.9(2)$ \\
$\mathrm{C}(1)-\mathrm{Na}(1)-\mathrm{C}(5)$ & $30.0(2)$ & $\mathrm{C}(1)-\mathrm{Na}(1)-\mathrm{N}(3)$ & $130.6(2)$ \\
$\mathrm{C}(2)-\mathrm{Na}(1)-\mathrm{C}(5)$ & $50.0(1)$ & $\mathrm{C}(2)-\mathrm{Na}(1)-\mathrm{N}(3)$ & $103.0(2)$ \\
$\mathrm{C}(3)-\mathrm{Na}(1)-\mathrm{C}(5)$ & $49.7(1)$ & $\mathrm{C}(3)-\mathrm{Na}(1)-\mathrm{N}(3)$ & $101.0(1)$ \\
$\mathrm{C}(4)-\mathrm{Na}(1)-\mathrm{C}(5)$ & $29.7(2)$ & $\mathrm{C}(4)-\mathrm{Na}(1)-\mathrm{N}(3)$ & $126.4(2)$ \\
$\mathrm{C}(1)-\mathrm{Na}(1)-\mathrm{N}(1)$ & $136.2(2)$ & $\mathrm{C}(5)-\mathrm{Na}(1)-\mathrm{N}(3)$ & $150.3(2)$ \\
$\mathrm{C}(2)-\mathrm{Na}(1)-\mathrm{N}(1)$ & $143.7(2)$ & $\mathrm{N}(1)-\mathrm{Na}(1)-\mathrm{N}(3)$ \\
$\mathrm{C}(3)-\mathrm{Na}(1)-\mathrm{N}(1)$ & $113.7(2)$ & $\mathrm{N}(2)-\mathrm{Na}(1)-\mathrm{N}(3)$ & $89.3(1)$ \\
$\mathrm{C}(4)-\mathrm{Na}(1)-\mathrm{N}(1)$ & $94.9(2)$ & & $86.4(1)$ \\
$\mathrm{C}(5)-\mathrm{Na}(1)-\mathrm{N}(1)$ & $106.4(2)$ & & \\
\hline & & & \\
& & & \\
& & & \\
& & & \\
& & & \\
& & &
\end{tabular}


Table 5

Selected bond lengths $(\dot{A})$ and angles $\left({ }^{\circ}\right)$ for 2

\begin{tabular}{|c|c|c|c|}
\hline $\mathrm{K}(1)-\mathrm{C}(10)$ & $3.059(2)$ & $\mathbf{K}(1)-C(20)$ & $3.104(2)$ \\
\hline$K(1)-C(30)$ & $3.055(2)$ & $K(1)-C(40)$ & $2.962(2)$ \\
\hline$K(1)-C(50)$ & $2.970(2)$ & $K(1)-N(1)$ & $2.879(2)$ \\
\hline$K(1)-N(2)$ & $2.974(2)$ & $K(1)-C(10 A)$ & $3.014(2)$ \\
\hline$K(1)-C(20 A)$ & $3.104(2)$ & $K(1)-C(30 A)$ & $3.102(2)$ \\
\hline$K(1)-C(40 A)$ & $3.020(2)$ & $K(1)-C(50 A)$ & $2.959(2)$ \\
\hline$C(10)-C(20)$ & $1.406(3)$ & $C(10)-C(50)$ & $1.409(3)$ \\
\hline$C(20)-C(30)$ & $1.415(3)$ & $C(30)-C(40)$ & $1.409(3)$ \\
\hline$C(40)-C(50)$ & $1.407(3)$ & & \\
\hline$C(10)-K(1)-C(20)$ & $26.4(1)$ & $C(10)-K(1)-C(30)$ & $43.9(1)$ \\
\hline$C(20)-K(1)-C(30)$ & $26.6(1)$ & $C(10)-K(1)-C(40)$ & $44.5(1)$ \\
\hline$C(20)-K(1)-C(40)$ & 44.1(1) & $C(30)-K(1)-C(40)$ & $27.0(1)$ \\
\hline$C(10)-K(1)-C(50)$ & $27.0(1)$ & $C(20)-K(1)-C(50)$ & $44.0(1)$ \\
\hline$C(30)-K(1)-C(50)$ & 44.4(1) & $C(40)-K(1)-C(50)$ & $27.4(1)$ \\
\hline $\mathrm{C}(10)-\mathrm{K}(1)-\mathrm{N}(1)$ & $119.2(1)$ & $C(20)-K(1)-N(1)$ & $93.1(1)$ \\
\hline$C(30)-K(1)-N(1)$ & $79.1(1)$ & $C(40)-K(1)-N(1)$ & $95.6(1)$ \\
\hline$C(50)-K(1)-N(1)$ & $121.8(1)$ & $C(10)-K(1)-N(2)$ & $93.7(1)$ \\
\hline$C(20)-K(1)-N(2)$ & $87.2(1)$ & $C(30)-K(1)-N(2)$ & $107.9(1)$ \\
\hline $\mathrm{C}(40)-\mathrm{K}(1)-\mathrm{N}(2)$ & $131.3(1)$ & $C(50)-K(1)-N(2)$ & $120.3(1)$ \\
\hline$N(1)-K(1)-N(2)$ & $84.3(1)$ & $C(10)-K(1)-C(10 A)$ & $141.7(1)$ \\
\hline$C(20)-K(1)-C(10 A)$ & $157.4(1)$ & $C(30)-K(1)-C(10 A)$ & $133.7(1)$ \\
\hline$C(40)-K(1)-C(10 A)$ & $113.3(1)$ & $C(50)-K(1)-C(10 A)$ & 117.1(1) \\
\hline$N(1)-K(1)-C(10 A)$ & $89.5(1)$ & $N(2)-K(1)-C(10 A)$ & $115.4(1)$ \\
\hline$C(10)-K(1)-C(20 A)$ & $116.2(1)$ & $C(20)-K(1)-C(20 A)$ & $138.7(1)$ \\
\hline$C(30)-K(1)-C(20 A)$ & $126.8(1)$ & $C(40)-K(1)-C(20 A)$ & $100.4(1)$ \\
\hline$C(50)-K(1)-C(20 A)$ & $94.7(1)$ & $N(1)-K(1)-C(20 A)$ & $114.5(1)$ \\
\hline$N(2)-K(1)-C(20 A)$ & $124.0(1)$ & $C(10 A)-K(1)-C(20 A)$ & $26.5(1)$ \\
\hline$C(10)-K(1)-C(30 A)$ & $109.0(1)$ & $C(20)-K(1)-C(30 A)$ & $135.4(1)$ \\
\hline$C(30)-K(1)-C(30 A)$ & $140.1(1)$ & $C(40)-K(1)-C(30 A)$ & $114.0(1)$ \\
\hline$C(50)-K(1)-C(30 A)$ & 97.3(1) & $N(1)-K(1)-C(30 A)$ & $131.0(1)$ \\
\hline $\mathrm{N}(2)-\mathrm{K}(1)-\mathrm{C}(30 \mathrm{~A})$ & $101.4(1)$ & $C(10 A)-K(1)-C(30 A)$ & $43.8(1)$ \\
\hline$C(20 A)-K(1)-C(30 A)$ & $26.4(1)$ & $C(10)-K(1)-C(40 A)$ & $126.5(1)$ \\
\hline$C(20)-K(1)-C(40 A)$ & $149.4(1)$ & $C(30)-K(1)-C(40 A)$ & $166.4(1)$ \\
\hline$C(40)-K(1)-C(40 A)$ & $140.5(1)$ & $C(50)-K(1)-C(40 A)$ & $122.2(1)$ \\
\hline$N(1)-K(1)-C(40 A)$ & $113.0(1)$ & $N(2)-K(1)-C(40 A)$ & $80.3(1)$ \\
\hline$C(10 A)-K(1)-C(40 A)$ & 44.4(1) & $C(20 A)-K(1)-C(40 A)$ & $43.7(1)$ \\
\hline$C(30 A)-K(1)-C(40 A)$ & $26.6(1)$ & $C(10)-K(1)-C(50 A)$ & $152.6(1)$ \\
\hline$C(20)-K(1)-C(50 A)$ & $175.0(1)$ & $C(30)-K(1)-C(50 A)$ & $158.2(1)$ \\
\hline$C(40)-K(1)-C(50 A)$ & $140.5(1)$ & $C(50)-K(1)-C(50 A)$ & $138.3(1)$ \\
\hline$N(1)-K(1)-C(50 A)$ & $88.2(1)$ & $N(2)-K(1)-C(50 A)$ & $88.2(1)$ \\
\hline$C(10 A)-K(1)-C(50 A)$ & $27.3(1)$ & $C(20 A)-K(1)-C(50 A)$ & $44.0(1)$ \\
\hline$C(30 A)-K(1)-C(50 A)$ & 44.1(1) & $C(40 A)-K(1)-C(50 A)$ & $27.2(1)$ \\
\hline $\mathbf{K}(1)-C(10)-K(1 A)$ & $132.8(1)$ & $K(1)-C(20)-K(1 A)$ & $127.4(1)$ \\
\hline$K(1)-C(30)-K(1 A)$ & $129.3(1)$ & $K(1)-C(40)-K(1 A)$ & $136.9(1)$ \\
\hline$K(1)-C(50)-K(1 A)$ & $139.6(1)$ & & \\
\hline
\end{tabular}

$\left[\mathrm{C}_{5} \mathrm{H}_{4}\left(\mathrm{SiMe}_{3}\right)\right] \mathrm{K}\left(150^{\circ}\right)$. This is presumably due to the additional coordination of two pyridine molecules to the potassium in 2. The $\mathrm{K}-\mathrm{Cp}$ (centre) distances (mean $2.79 \AA)$ are similar to the corresponding distances found in $\left[\left(\mathrm{C}_{5} \mathrm{H}_{4}\left(\mathrm{SiMe}_{3}\right)\right] \mathrm{K}(2.78\right.$ $\AA)$ and in the monomeric $\left[\mathrm{C}_{5}\left(\mathrm{CH}_{2} \mathrm{C}_{6} \mathrm{H}_{5}\right)_{5}\right] \mathrm{K} \cdot 3 \mathrm{THF}(2.79 \AA)$. The $\mathrm{K}-\mathrm{C}$ distances (mean $3.03 \AA$ ) are comparable to those in $\left[\left(\mathrm{C}_{5} \mathrm{H}_{4}\left(\mathrm{SiMe}_{3}\right)\right] \mathrm{K}(3.03 \AA)\right.$ and in 

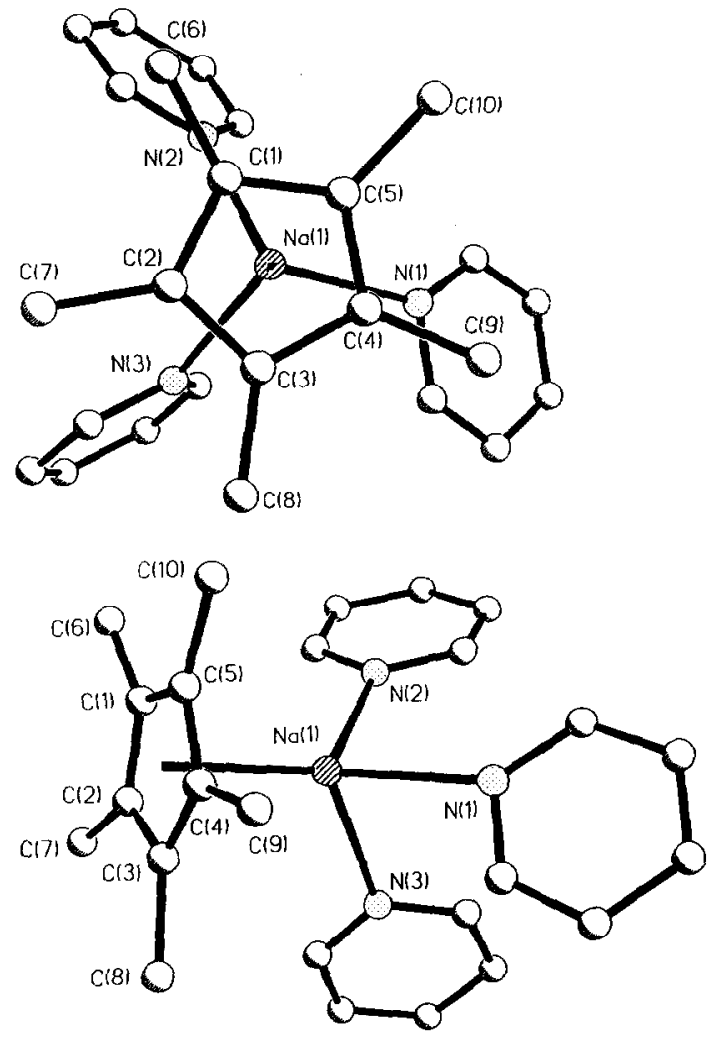

Fig. 1. Two views of the molecular structure of the $\left[\mathrm{C}_{5} \mathrm{Me}_{5}\right] \mathrm{Na} \cdot 3 \mathrm{py}$ monomer of 1 .
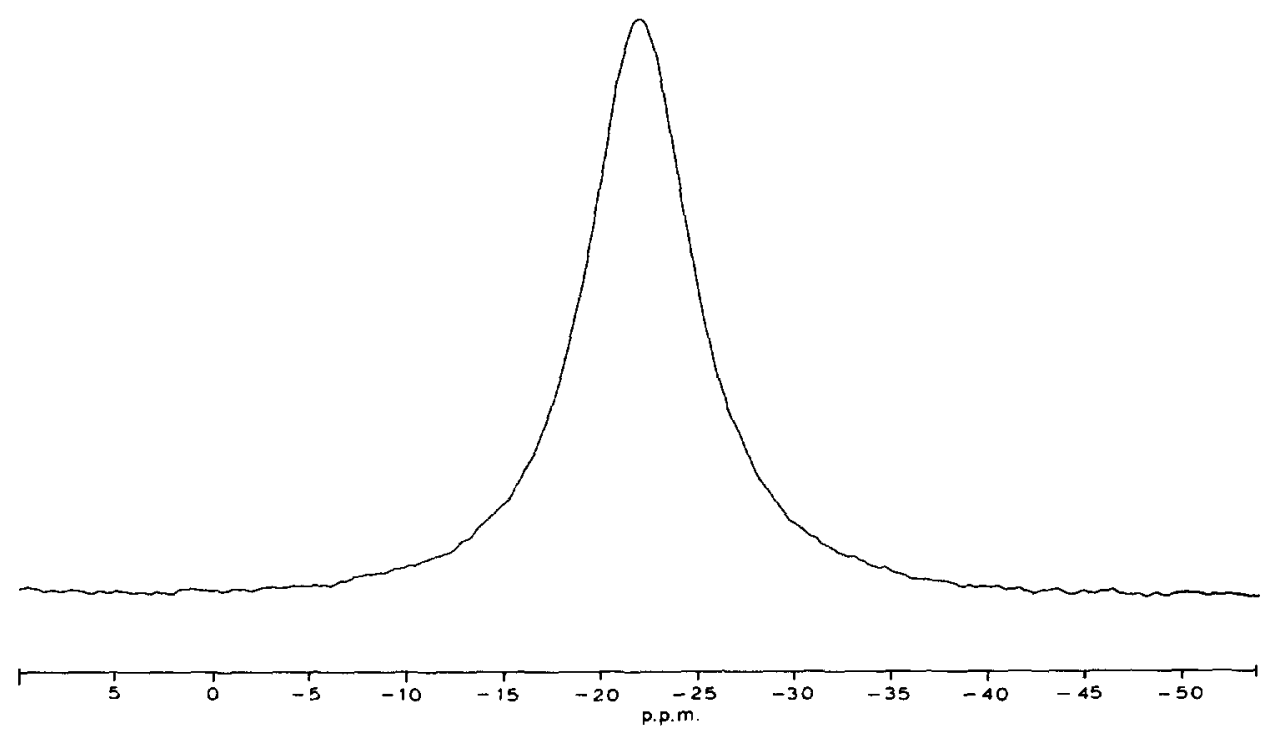

Fig. 2. Solution ${ }^{23} \mathrm{Na}-\mathrm{NMR}$ spectrum of $\left(\mathrm{C}_{5} \mathrm{Me}_{5}\right) \mathrm{Na}$ in $\mathrm{C}_{5} \mathrm{D}_{5} \mathrm{~N}$ at $25^{\circ} \mathrm{C}$. 


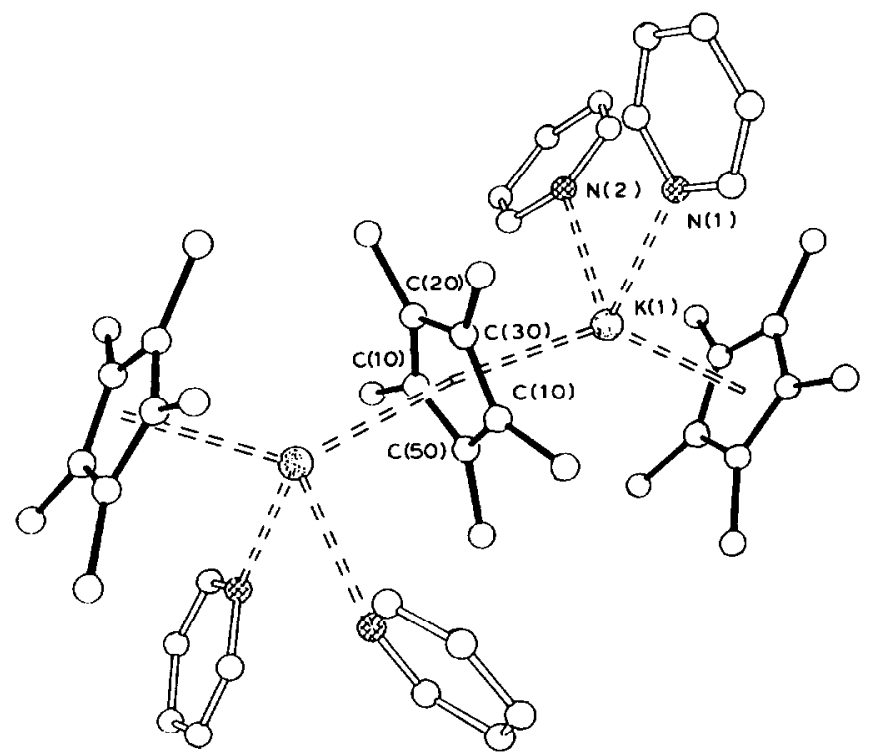

Fig. 3. The structure of the zig-zag $\left[\left(\mathrm{C}_{5} \mathrm{Me}_{5}\right) \mathrm{K} \cdot 2 \mathrm{py}\right]_{\infty}$ chain of 2.

$\left[\mathrm{C}_{5}\left(\mathrm{CH}_{2} \mathrm{C}_{6} \mathrm{H}_{5}\right)_{5}\right] \mathrm{K} \cdot 3 \mathrm{THF}(3.04 \AA)$. The $\mathrm{K}-\mathrm{N}$ distances in 2 are $2.879(2)$ and $2.974(2) \AA$.

The change in structure from monomeric to polymeric for 1 and 2 and the increasing coordination number at the metal can be accounted for in terms of the increasing alkali metal radius. Consequently by substituting the sodium in the $\left(\mathrm{C}_{5} \mathrm{Me}_{5}\right) \mathrm{Na} \cdot 3$ py monomer with a larger and less polarizing potassium would result in decreased steric crowding and weaker $\mathrm{Cp}^{\star-} \mathrm{M}^{+}$ion pair and cation-ligand interactions. Thus removal of one pyridine ligand from a $\left(\mathrm{C}_{5} \mathrm{Me}_{5}\right) \mathrm{K} \cdot 3$ py monomer would allow a second $\mathrm{Cp}^{\star}$-ring to coordinate to the potassium with resulting chain formation.

\section{Experimental}

All syntheses were carried out under pure nitrogen in dried and freshly distilled solvents. Mass spectrum: Finnigan 8230. NMR-spectra in $\mathrm{C}_{5} \mathrm{D}_{5} \mathrm{~N}$ (standards): ${ }^{1} \mathrm{H}$, ${ }^{13} \mathrm{C}\left(\mathrm{TMS}_{\text {ext. }}\right) ;{ }^{23} \mathrm{Na}\left(\mathrm{NaCl}_{\text {sat. }}\right.$ in $\mathrm{D}_{2} \mathrm{O}, \Delta \nu_{1 / 2}=13 \mathrm{~Hz}$ at $\left.25^{\circ} \mathrm{C}\right)\left[11^{*}\right]$ : Bruker $\mathrm{AM}$ 250.

\section{Sodium pentamethylcyclopentadiene}

Sodium metal and a solution of $(1.0 \mathrm{~g}, 43 \mathrm{mmol})$ pentamethylcyclopentadiene $(7.0 \mathrm{~g}, 51 \mathrm{mmol})$, in toluene $(80 \mathrm{ml})$ were vigorously stirred together for $3 \mathrm{~d}$ under reflux. After filtration and washing with toluene $(30 \mathrm{ml})$ the residue was extracted with tetrahydrofuran. Removal of the solvent gave $6.5 \mathrm{~g}$ (95\%) of sodium pentamethylcyclopentadiene as a white powder; m.p. $>250^{\circ} \mathrm{C}$. ${ }^{1} \mathrm{H}$ NMR $\left(\mathrm{C}_{5} \mathrm{D}_{5} \mathrm{~N}\right)$ : $\delta(\mathrm{Me})=2.41 \mathrm{ppm} ;{ }^{13} \mathrm{C}$ NMR $\left(\mathrm{C}_{5} \mathrm{D}_{5} \mathrm{~N}\right): \delta(\mathrm{Me})=12.4,\left(\mathrm{C}_{5}\right) 105.5 \mathrm{ppm} ;{ }^{23} \mathrm{Na} \mathrm{NMR}$ $\left(\mathrm{C}_{5} \mathrm{D}_{5} \mathrm{~N}\right): \delta(\mathrm{Na})=-22.0 \mathrm{ppm}$. 


\section{Potassium pentamethylcyclopentadiene}

A mixture of potassium hydride $(1.0 \mathrm{~g}, 25 \mathrm{mmol})$, pentamethylcyclopentadiene $(4.0 \mathrm{~g}, 29 \mathrm{mmol})$, and tetrahydrofuran $(40 \mathrm{ml})$ was stirred for $14 \mathrm{~h}$ at room temperature then filtered. Washing of the residue with tetrahydrofuran $(30 \mathrm{ml})$ and drying under vacuum gave $4.1 \mathrm{~g}(95 \%)$ of potassium pentamethylcyclopentadiene as a white powder; m.p. $>250{ }^{\circ} \mathrm{C} .{ }^{1} \mathrm{H}$ NMR $\left(\mathrm{C}_{5} \mathrm{D}_{5} \mathrm{~N}\right): \delta(\mathrm{Me})=2.23 \mathrm{ppm} ;{ }^{13} \mathrm{C} \mathrm{NMR}$ $\left(\mathrm{C}_{5} \mathrm{D}_{5} \mathrm{~N}\right): \delta(\mathrm{Me})=11.91,\left(\mathrm{C}_{5}\right) 106.1 \mathrm{ppm}$; MS (FD): $m / z$ (ion) $=213\left(M+\mathrm{K}^{+}\right)$, $39\left(\mathrm{~K}^{+}\right)$.

\section{Crystal growth and $X$-ray measurements of 1 and 2}

Crystals of 1 and 2 were grown during $1-2$ weeks by slowly condensing the solvent off a pyridine solution of $\left(\mathrm{C}_{5} \mathrm{Me}_{5}\right) \mathrm{Na}$ or $\left(\mathrm{C}_{5} \mathrm{Me}_{5}\right) \mathrm{K}$.

Both structures were measured on a Stoe-Siemens four-circle diffractometer with graphite-monochromated Mo- $K_{\alpha}$ radiation $(\lambda=71.069 \mathrm{pm})$. Data were collected at $-120^{\circ} \mathrm{C}$ with a profile fitting method [14], $2 \theta=8-45^{\circ}$ (1) and $8-50^{\circ}$ (2). The structures were solved by Patterson methods and refined by full-matrix least squares, with all non-hydrogen atoms anisotropic. All hydrogen atoms were found by difference Fourier synthesis and refined isotropically. A riding model was employed for the hydrogen atoms with an idealized bond length of $96 \mathrm{pm}$. A semi-empirical absorption correction was applied in both cases. All calculations were performed with SHELX-76 and SHELXS-86 $[15,16]$.

Supplementary material available. Tables of intensity measurement and refinement parameters, bond lengths, bond angles, anisotropic displacement parameters, $H$ atom coordinates and isotropic displacement parameters and a list of observed and calculated structural factors are available from the authors. Further details of the X-ray structural investigation can be obtained from the Fachinformationszentrum Karlsruhe, Gesellschaft für wissenschaftlich-technische Information $\mathrm{mbH}$, D-7514 Eggenstein-Leopoldshafen 2, under quotation of the deposit number CSD54823, the authors and the publication.

\section{Acknowledgements}

We thank Dr. Már Björgvinsson for valuable comments. We thank the LeibnizProgramm der Deutschen Forschungsgemeinschaft and the Fonds der Chemischen Industrie for operating grants, and the Fonds der Chemischen Industrie for an award of a scholarship to G.R.

\section{References}

1 P. Jutzi, Comments Inorg. Chem. 6 (1987) 123.

2 P. Jutzi, D. Kanne, C. Krüger, Angew. Chem. 98 (1986) 163; Angew. Chem. Int. Ed. Engl. 25 (1986) 164; O.T. Beachley, Jr., M.R. Churchill, J.C. Fettinger, J.C. Pazik, L. Victoriano, J. Am. Chem. Soc. 108 (1986) 4666; T.F. Berlitz, H. Sinning, J. Lorberth, U. Müller, Z. Naturforsch. B43 (1988) 744.

3 M.F. Lappert, A. Singh, L.M. Engelhardt, A.H. White, J. Organomet. Chem. 262 (1984) 271.

4 P. Jutzi, E. Schlüter, S. Pohl, W. Saak, Chem. Ber. 118 (1985) 1959.

5 P. Jutzi, E. Schlüter, C. Kriger, S. Pohl, Angew. Chem. 95 (1983) 1015; Angew. Chem. Int. Ed. Engl. 22 (1983) 994.

6 A. Hammel, Dissertation 1989. Universität Stuttgart.

7 T. Aoyagi, H.M.M. Shearer, K. Wade, G. Whitehead, J. Organomet. Chem. 175 (1979) 21. 
8 R.D. Rogers, J.L. Atwood, M.D. Rausch, D.W. Macomber, W.P. Hart, J. Organomet. Chem. 238 (1982) 79.

9 P. Jutzi, W. Leffers, B. Hampel, S. Pohl, W. Saak, Angew. Chem. 99 (1987) 563; Angew. Chem. Int. Ed. Engl. 26 (1987) 583.

10 J. Lorberth, S.-H. Shin, S. Wocadio, W. Massa, Angew. Chem. 101 (1989) 793; Angew. Chem. Int. Ed. Engl. 28 (1989) 735.

11 For a $1 M$ sodium chloride solution in $\mathrm{D}_{2} \mathrm{O}$ we measured an upfield shift of $0.65 \mathrm{ppm}\left(\Delta \nu_{1 / 2}=12\right.$ IIz) relative to saturated sodium chloride solution.

12 G.J. Templeman, A.L. Van Geet, J. Am. Chem. Soc. 94 (1972) 5578.

13 B. Lindmann, S. Forsen, in R.K. Harris, B.E. Mann (Eds.) NMR and the Periodic Table, Academic Press, 1978. London'New York 'San Francisco, Ch. 6, p. 131.

14 W. Clegg, Acta Crystallogr. A, 37 (1981) 22.

15 G.M. Sheldrick, SHELX-76, Universität Göttingen, modified by the author.

16 G.M. Sheldrick, Acta Crystallogr. A, 46 (1990) 467. 